$p=0.02$, respectively); however, among MSM, age was no longer statistically significant ( $62.8 \%$ vs. $70.8 \%, p=0.25$, respectively).

Conclusions A substantial proportion of persons newly diagnosed with HIV were also recently infected with syphilis, with young age strongly associated with ES among non-MSM. In addition to MSM, women and MSW under 30 years old may be appropriate foci for targeted control.

\section{P3.213 SEROPREVALENCE OF HUMAN IMMUNODEFICIENCY VIRUS (HIV) INFECTION AMONG TUBERCULOSIS PATIENTS ATTENDING TB/DOTS CENTRE IN NNEWI}

doi:10.1136/sextrans-2013-051184.0670

'S O Kalu, ${ }^{2} \mathrm{P}$ U Ele, ${ }^{3 \mathrm{R}} 0$ Okonkwo, ${ }^{3} \mathrm{C}$ N Ogbuagu, ${ }^{3} \mathrm{~B} 0$ Oluboyo. 'of Human Virology Dept., Nnamdi Azikiwe University Teaching Hospital Nnewi, Nnewi, Nigeria; ${ }^{2}$ nstitute of Human Virology Dept., Nnamdi Azikiwe University Teaching Hospital Nnewi, Nnewi, Nigeria; ' ${ }^{3}$ Medical Microbiology/Parasitology Dept., Nnamdi Azikiwe University Teaching Hospital Nnewi, Nnewi, Nigeria

Background TB and HIV co-epidemic is a major public health problem in many parts of the world, particularly in developing counties. This study was a prospective cohort design to determine the seroprevalence of HIV infection among tuberculosis patients attending TB/Directly Observed Treatment Short-course (DOTS) centre in a tertiary hospital in Nnewi, Nigeria

Methods TB diagnosis was based on combined evaluations of clinical, radiological and laboratory features of the patients with the protocol established by the National Tuberculosis Control Program (NTBCP). Laboratory diagnosis of HIV infection was based on rapid HIV test kits according to the national HIV testing algorithm.

Results Of the 1356 tuberculosis patients tested, 404/1356 (29.9\%) were positive to the HIV antibodies. The prevalence was higher in females (15.6\%) compared to males (14.2\%). The prevalence of HIV in 49 years of age or less population was 15.6 times (28.0\%) higher compared to 50 years and older (1.8\%). 823 out of 1356 (60.6\%) were Smear Positive TB (SPTB). Extra-Pulmonary Tuberculosis (EPTB) 89/404 (22\%) and Smear Negative TB (SNTB) 326/404 $(58.7 \%)$ were frequently associated with HIV/TB co-infection.

Conclusion Our results indicate that the prevalence of HIV/TB co-infection in Nnewi, Nigeria deserves special attention, screening of HIV among TB populations should be performed as this would assist in the treatment of both diseases.

\section{P3.214 HIV PREVALENCE TREND IN THE CONFLICT TO POST- CONFLICT TRANSITION PERIOD IN GULU DISTRICT, NORTHERN UGANDA}

doi:10.1136/sextrans-2013-051184.0671

'E Ochola, 'P Bayo, 'M D Ogwang, ${ }^{2} \mathrm{~A}$ Opio, ${ }^{3} \mathrm{M}$ Fabiani. 'St. Mary's Hospital Lacor, Gulu, Uganda; ${ }^{2}$ Uganda Ministry of Health, Kampala, Uganda; ${ }^{3}$ Italian National Institute for health, Epidemiology and Communicable Disease Unit, Rome, Italy

Background Since 1986, North Uganda has been affected by civil strife and most of its population have been displaced in protected camps. However, since 2007, the increased security conditions have allowed many people to leave the camps and return to their villages. This study aims at estimating the HIV prevalence trend among pregnant women in Gulu district in the conflict (2005-2006) to post-conflict (2007-2010) transition period.

Methods In 2005-2006 and 2007-2010, a total of 2318 and 25,924 ANC attendees of the St. Mary's Hospital Lacor, respectively, were anonymously tested for HIV within the national sentinel surveillance system. Differences in HIV prevalence by testing period and displacement status were evaluated using the chi-square test.

Results The overall HIV prevalence in 2005-2006 was $11.0 \%$ compared with $9.9 \%$ in $2007-2010$ ( $\mathrm{P}=0.074$ ). In both periods, as previously found, prevalence among internally displaced women
(IDW) was lower than prevalence among women living outside camps. However, the difference in prevalence between these two groups decreased in the transition period. In fact, while the prevalence remained quite stable among IDW $(9.2 \%$ in $2005-2006 \mathrm{com}-$ pared with $8.3 \%$ in $2009-2010 ; \mathrm{P}=0.370$ ), it significantly decreased among women living outside camps (12.6\% in 2005-2006 compared with $10.4 \%$ in $2009-2010 ; \mathrm{P}=0.020$ ), mostly reflecting the population movements occurred since 2007 (IDW were $45.0 \%$ of the ANC attendees in 2005-2006 compared with $27.5 \%$ in $2009-2010$; $\mathrm{P}<0.001)$.

Conclusions The HIV prevalence in Gulu district is still high compared with the rest of Uganda. It remained quite stable, thus suggesting that no HIV-related behavioural changes in the post-conflict period have occurred or that their effects are not yet observable. However, the reduced difference in HIV prevalence between IDW and women living outside of protected camps suggests that the HIV epidemiological profile in this district is changing, mainly as a result of the post-conflict population movements

\section{P3.215 HIV/AIDS COINFECTION WITH THE HEPATITIS B AND C VIRUSES IN BRAZIL}

doi:10.1136/sextrans-2013-051184.0672

${ }^{1}$ S B Oliveira, ${ }^{2}$ E M Hamann, ${ }^{3} \mathrm{~L}$ A F Amorim. ${ }^{1}$ Departamento de DST, AIDS E Hepatites Virais, Brasilia, Brazil; ${ }^{2}$ Universidade de Brasilia, Brasilia, Brazil; ${ }^{3}$ Universidade Federal da Bahia, Brasilia, Brazil

Background Hepatitis $\mathrm{B}$ and $\mathrm{C}$ viruses and the HIV virus share the most important forms of transmission. Infections by these viruses present a dynamic interaction, amplifying each other and leading to greater morbidity and mortality. The objective of this study is to estimate the prevalence and to describe the epidemiological profile of individuals coinfected with HIV/HBV and HIV/ $\mathrm{HCV}$ in Brazil.

Methods AIDS cases were obtained from the "Sistema de Informação de Agravos de Notificação" (Sinan) and the "Sistema de Controle de Exames Laboratoriais" (Siscel). Coinfection was identified through probabilistic record linkage of the AIDS cases with hepatitis viral ( $\mathrm{B}$ and $\mathrm{C}$ ) from Sinan's notifications. The probabilistic records linkages were performed using the RecLink III software.

Results Between 1999 and 2010, 370,672 AIDS cases were reported, of which $3,724(1.0 \%)$ were identified as HIV/HBV coinfections and $5,932(1.6 \%)$ as HIV/HCV coinfections. The chance of coinfection increases with age, it is 3 times higher in aged 45 and older individuals coinfected with HBV than patients aged 24 and younger; the chance is 12 times higher among those coinfected with HCV. The chance for coinfections increases 2- to 6-fold for HBV and $\mathrm{HCV}$, respectively, for the "injecting drugs users" (IDU) category compared to sexual exposure.

Conclusions The IDU category is one of the main forms of HCV and HIV transmissions, which may explain the higher chance of coinfection in this category. This study permitted an important evaluation of HBV/HIV and HCV/HIV coinfections in Brazil by the use of reported cases, without the need to conduct seroprevalence research.

\section{P3.216 HIV AND VIRAL HEPATITIS (B,D,C) CO-INFECTION, GENOTYPING, EPIDEMIOLOGICAL PROFILE IN WEST OF TEHRAN}

doi:10.1136/sextrans-2013-051184.0673

S Sali, 'M Nikbin, 'D Yadegarinia, ${ }^{2} \mathrm{M}$ Hesseinifar, ${ }^{2} \mathrm{M}$ Shahmuradi. ${ }^{1}$ Infectious Diseases and Tropical Medicine Research Center, Faculty of Medicine Shahid Beheshti University of Medical Sciences, Tehran, Iran; ${ }^{2}$ West Health Center, Tehran, Iran

Background/Aims In HIV infected patients, HBV, HDV and HCV co-infections have important implications for their clinical course 
and therapeutic management. There is intermediate prevalence of HBV and low prevalence of HIV and HCV in Iran. However, there is little information regarding the number of co-infections of viral hepatitis. The aim of present is study is to establish prevalence of HBV, HDV and HCV co-infection among HIV positive Iranian patients.

Materials and Methods The design of study was cross-sectional during 2010 to 2012. Patients having HIV with co-infection of HBV, HDV or HCV visiting Tehran West Health Centre were enrolled. Serological HBV (HBS Ag, HBC Ab), HDV Ab and HCV Ab were determined in a sub-group of $200 \mathrm{HIV}$ positive patients. HCV RNA PCR(Viral load and genotyping) was determined for all HCV Ab positive patients. Also, HDV Ab was determined for all HBS or HBC Ab positive patients.

Results A total of 200 patients (151 male and 49 female) with a mean age of 33 (2 to 66 years) were evaluated. The prevalence of HBS Ag and HBC Ab was 12\% (21/177) and 24\% (43/177), respectively. HCV Ab was detected in $71 \%(123 / 173)$ out of which $90 \%$ (110/123) were PCR positive with 1b Genotype being the most prominent case. For $43 \mathrm{HBS} \mathrm{Ag}+$ patients, HDV Ab was carried out, 5 of whom were positive and 3 were triple HCV, HBV and HDV coinfection.

Conclusions A high rate of $\mathrm{HIV} / \mathrm{HBV}, \mathrm{HCV}$ co-infection observed in the present study, indicates the need for routine screening of HIV infected patients for viral hepatitis B and C. Such screening could lead into the required treatment for these patients.

\section{P3.217 INTERNATIONAL COMPARISON OF RECENT TRENDS IN THE RATES OF HIV DIAGNOSES AMONG MEN WHO HAVE SEX WITH MEN (MSM)}

doi:10.1136/sextrans-2013-051184.0674

T Foster, N Dickson, P Saxton. University of Otago, Dunedin, New Zealand

Background After a rise in the early 2000s in the number of new HIV diagnoses among MSM in New Zealand, also witnessed in many developed countries, in 2011 the number dropped by $34 \%$ compared to 2010 . To assess relative progress on control we compare trends in HIV diagnosis rates among MSM in developed countries with similarly mature epidemics.

Methods We obtained data on annual HIV diagnoses among MSM between 2003-2011 from 17 developed countries (Australia, Belgium, Canada, Denmark, Finland, France, Iceland, Ireland, Netherlands, New Zealand, Norway, Spain, Sweden, Switzerland, Germany, UK, US). We reallocated those with unknown means of infection according to the countries' pattern of known causes, and used countries' adjustment for delayed reporting where available. The diagnosis rate was derived using the population of men aged 15-64.

Results

- New Zealand has low rates compared to most countries of Western Europe, North America and Australia, and are comparable with those of Scandinavia

- All counties except New Zealand, Iceland and Canada, experienced a slight overall rise in diagnosis rates in the period 2003-2011

- Over the past four years there has been a:

- Slight trend upwards in UK, Belgium, France, Australia, Ireland

- No clear trend in Spain, Canada, Germany, Denmark, Norway

- Slight trend downwards in New Zealand, the Netherlands, Sweden, Finland, and possibly Iceland

- Clear trend downwards in Switzerland.

Conclusions New Zealand has a low rate of HIV diagnoses, relative to many other developed countries. Our drop in 2011 HIV is encouraging but not unique. Limitations of this study are that the data are of diagnosis not infection rates, are influenced by patterns of testing, immigration and emigration, and dual modes of transmission, and the proportion who are MSM may vary between countries. Factors relating to recent trends should be explored.

\section{P3.218 INCREASING TREND OF HIV/AIDS AMONG ARAB AND JEWISH MALES IN ISRAEL, 1986-2010}

doi:10.1136/sextrans-2013-051184.0675

'Z Mor, ${ }^{2} \mathrm{E}$ Grayeb, ${ }^{3} \mathrm{~A}$ Beany, ${ }^{4} \mathrm{G}$ Grotto. 'Ministry of Health, Ramla, Israel; ${ }^{2}$ Hadassah Medical Center, Jerusalem, Israel; ${ }^{3} B n a i$ Zion Medical Center, Haifa, Israel; ${ }^{4}$ Ministry of Health, Jerusalem, Israel

Background HIV/AIDS-burden in Israel is low ( $~ 6$ cases:100,000 population), while sub-populations characterised by high-risk behaviours are affected disproportionally. This study aims to compare HIV/AIDS-burden in males between Israeli Jews and Arabs, which are the biggest monitory in Israel.

Methods The National HIV/AIDS Registry (NHAR) was the source for HIV/AIDS-infection records, while the Israeli Central Bureau of Statistics was used to determine groups-specific disease rates.

Results Between 1986 and 2010, 3,499 HIV/AIDS-infected males were reported: 3,369 (96.3\%) Jews and 130 (3.7\%) Arabs, in an average annual incidence of 5.5 and 0.8 per 100,000 populations, respectively, $p=0.05$. Of all Jews, 1,018 (29.9\%) were born in Ethiopia, while 2,389 were Jews who were not Ethiopian-born (JNE). Most Arabs ( $\mathrm{N}=99$, 74.8\%) were Muslims, followed by 21 (16.2\%) Christians and 13 (10\%) Druze. AIDS rather than HIV upon reporting was diagnosed in $568(23.8 \%)$ of JNE and $31(23.8 \%)$ of the Arabs, $p=1$. The most affected age-group among JNE was 25-34 and in Arabs 20-24, and the respective cumulative death rates were $24.9 \%(\mathrm{~N}=594)$ and $32.5 \%$ $(\mathrm{N}=40), \mathrm{p}=0.1$. The point-prevalence in 2010 was 58.4 and 11.4 per 100,000 for JNE and Arabs, and in adults aged 15-49, was 98.0 and 20.4 per 100,000, respectively. In Muslims, Christians and Druze, the point-prevalence was 4.2,11.2 and 7.1 per 100,000; and in adults aged 15-49 was 20.4, 52.6 and 21.6, respectively.

The most common risk-groups among JNE was MSM (N =1,223, $51.2 \%$ ), followed by IVDU ( $\mathrm{N}=661,27.7 \%)$; while among Arabs was MSM ( $\mathrm{N}=63,48.1 \%)$, followed by heterosexuals $(\mathrm{N}=36$, $27.3 \%)$.

Conclusions HIV/AIDS-burden in Arab males was significantly lower than in Jews. Among Arab-males, HIV/AIDS-burden was highest in Christians than in Druze and Muslims. The proportion of MSM of all males, regardless of their religion is increasing. Interventions aiming to prevent further HIV-transmission should address cultural, linguistic and behavioural characteristics.

\section{P3.219 A 20-YEARS RETROSPECTIVE COHORT STUDY OF HIV SITUATION AMONG HILL TRIBE VULNERABLE POPULATION THAILAND}

doi:10.1136/sextrans-2013-051184.0676

A Tawatchai. School of Health Science, Mae Fah Luang University, Chiang Rai, Thailand

Thailand had been reported as the highest HIV/AIDS epidemic area. Most of HIV/AIDS had been reported from the north of Thailand. Northern Thailand is the favourite living places of hill tribe people who migrated from the south of China through Myanmar last 150 years ago. 600,000 people were living in these areas with differences of culture and life styles. The objective aimed to investigate the situation of HIV/AIDS among hill tribe marginalised and vulnerable population.

Methods The retrospective cohort study was conducted. The systematic data extraction from the medical records in 16 hospitals in northern Thailand during 1990-2010 was performed. The six main hill tribe people: Akha, Lau, Karen, Yao, Kmong, and Lisu were the target population. Chi square test was analysed. 